\title{
ATC Malachlorite® for treatment of patients with acute Plasmodium falciparum infection: A pilot study incorporating 500 patients in the rural area of Cameroon
}

\author{
Enno Freye ${ }^{1 *}$, Hans-Peter Strobel ${ }^{2}$ and Olivia M. Weber ${ }^{3}$ \\ ${ }^{1}$ University Clinics of Düsseldorf, Germany. \\ 2 Department of Energy Development Davos-Platz, Switzerland \\ ${ }^{3}$ Center of Novel Therapeutic Strategies (NTS), Luzern, Switzerland.
}

Publication history: Received on 17 January 2018; revised on 27 January 2018; accepted on 07 February 2018

https://doi.org/10.30574/gscbps.2018.2.2.0004

\begin{abstract}
Malaria is one of the most widespread infectious diseases with over 2 million deaths per year in Central Africa. Since new drug combinations are characterized by the development of tolerance as well as serious side effect there is an increasing demand for a new medication. We therefore set out to study the efficacy of a sublingual tablet with a new mode of action consisting of sodium chlorite $\left(\mathrm{NaClO}_{2}\right)$ plus citric acid and an artimisin extract. By adding sodium bicarbonate, an effervescent composition is attained, which when being exposed to saline, chlorine dioxide $\left(\mathrm{ClO}_{2}\right)$ the active ingredient is being released. Being a potent oxygen radical, it attaches to the layer of a trophozoite resulting in the disintegration and its demise. This reaction is terminated within one hour and $\mathrm{ClO}_{2}$ dissolves into the two end products, sodium chloride (table salt) and water. Following informed consent 500 patients (240 male, 260 female mean age $27+/-19$ SD) within the rural area of Cameroon and demonstrating acute symptoms of malaria infection, were given 5 sublingual tablets of ACT Malachlorite 3 times per day at an 8 hour interval. The medication was able to reverse acute symptoms of malaria within the first 2 days. Using thick blood films, trophozoite count under the microscope demonstrated a highly significant reduction $(\mathrm{p}<0.001)$ of count of Plasmodium falciparum. From a mean of $6430(+/-$ 816 SD) trophozoites per mm3, prarasitemia dropped to a mean of 4932 (+/- 842 SD) on day two and further declined to 1454 (+/- 401 SD; p < 0.001) on day four. No trophozoites could be detected in the blood on day six. Side effects were reported from the intestinal tract consisting of bloating and cramping while the majority of patients complained of the pungent chlorine taste as being unpleasant. The new ATC Malachlorite $®$ tablet presents a promising new approach in malaria treatment with a differentmode of action than all present antimalarial agents.
\end{abstract}

Keywords: Malaria treatment; Plasmodium falciparum; Chlorine dioxide; Pilot study Cameroon

\section{Introduction}

Malaria affects about a hundred countries in the world, particularly the disadvantaged tropical areas of Africa, Asia and Latin America. Africa is by far the most affected continent with $90 \%$ of malaria cases in tropical areas. Malaria is a disease caused by parasites of the plasmodium type, which according to WHO, causes around 1 million deaths per year worldwide. About $40 \%$ of the world population is exposed to the disease and 500 million clinical cases occur each year. On the other hand, Europe is experiencing cases of so-called imported malaria. Especially countries like Britain and France show the highest number of imported malaria as reported recently [1]. The situation is particularly worrying in that for several years the parasites develop resistance to many commonly used anti-malarial agents while in addition, mosquitoes demonstrate an increase of resistance to the present available insecticides. So far, no vaccine is available for this life-threatening disease resulting in every new exposure into a full-blown course of the malaria disease. While 
it is generally accepted that malaria is one of the most widespread infectious diseases representing over 2 million deaths per year in Central Africa and although every medical and hygienic effort is undertaken to reduce the number, reports of WHO show a steady increase in the number of infections as well as the development of tolerance to conventional drugs such as resochine (Chloroquine $\AA$ ), the antibiotic tetracycline Doxycycline $\AA$ or the combination Malarone $\AA$ (Atovaquon-Proguanil), all of which are not only characterized by resistance development but also of severe side effects [2]. Although the latest generation of agents in the treatment of malaria such as Lariam $₫$ (a chinine derivative) showed little resistance development they are however characterized by serious side effects. And while treatment is readily available in large cities where hospital doctors as well as medical staff are able to treat acute symptoms of malaria, the inhabitants of suburban and rural areas lack such advanced care [3]. It is because in such remote areas, the general medical practitioner (GP) is only visited by patients who demonstrate acute symptoms of Plasmodium falciparum infection, having reached a critical state. Especially, development of tolerance to conventional medication presents a problem in treatment of an acute infection when using the ACT (additional combination therapy) consisting of a two-drug approach, one of which is always an artemisinin derivative [2-4].

In spite of such new strategies in the treatment of malaria, there is an ongoing demand for alternative therapeutic agents which do not present the problem of tolerance development. Therefore, the objective of the study was to ascertain, that this newly formulated effervescent tablet is easily accessible to patients in urban areas, demonstrates a high therapeutic efficacy, is easily administered to patients, and does not demonstrate the usual side effects usually seen with the conventional medications.

\section{Material and methods}

\subsection{Composition of ATC Malachlorite $®$ - mode of action}

We therefore set out to study the efficacy of a totally new medication which so far had only been used for the manufacture of safe drinking water in a number of small city water plants in rural areas and for trekker and boot camping and which already is in practical use for the past 10 years. This compound by the name of sodium chlorite $\left(\mathrm{NaClO}_{2}\right)$, when getting into contact with an acidic acid, dissolves into chlorine dioxide $\left(\mathrm{CLO}_{2}\right)$ which by itself acts as a potent oxygen radical releaser. When it attaches to the layer of a trophozoite, it induces the disintegration of the outside covering of the protozoon and its immediate demise. By adding sodium bicarbonate an effervescent composition was developed, which when being exposed to water or to saline, results into the release of $\mathrm{ClO}_{2}$ being the active ingredient. This chlorine dioxide is readily taken up by the mucous membranes of the oral cavity and after entering the blood cycle it attaches to any kind of preexisting trophozoite. This reaction is terminated within one hour after chlorine dioxide has reached the blood where it dissolves into the two end products, sodium chloride ( $\mathrm{NaCl}$ or table salt) and water, showing the following chemical reaction:

$$
5 \mathrm{NaClO}_{2}+\mathrm{C}_{6} \mathrm{H}_{8} \mathrm{O}_{7}=4 \mathrm{ClO}_{2}+5 \mathrm{NaCl}+2 \mathrm{H}_{2} \mathrm{O}
$$

Thus, the compressed tablet, aside from containing the prodrug sodium chlorite $\left(\mathrm{NaClO}_{2}\right)$ and the additive citric acid $\left(\mathrm{C}_{6} \mathrm{H}_{8} \mathrm{O}_{7}\right)$ which later serves as the ignition key for the production of $\mathrm{ClO}_{2}$, are kept in a compressed tablet to which artimisin extract was added. This addition deemed necessary being an agent already known for a long time in traditional Chinese medicine as an efficient antimalarial agent but also since WHO demands that any new agent contains parts of artimisin or its active ingredient artemisinin [5]. All other compositions added to the tablet, however, are inert and are used solely either to increase solubilization, the reabsorption through the mucous membranes or to increase tastiness (table 1).

The gas chlorine dioxide $\left(\mathrm{ClO}_{2}\right)$ is the active compound, being reabsorbed through the mucous membranes and/or the intestinal lining inducing a sufficiently high serum level. Because of the negative charge of chlorine dioxide, the agent is attracted by the positive charge of any kind of parasite, and by closely attaching to the layer to the Plasmodium falciparum it releases a highly aggressive oxygen radical $\left(\mathrm{O}_{2}^{-}\right)$. This radical in order for the sake of stability, tears out electrons from the outer lining of the parasite resulting in the disintegration of its layer which is followed by its demise.

All of the active compounds of the tablets were crushed into tiny pieces of $250 \mathrm{pm}$ in diameter. Thereafter, the raw powder was added to the auxiliaries and the whole was compressed into tablets of $0.7 \mathrm{~g}$ (table 1). These tablets were given to patients having symptoms of an acute malaria infection characterized by acute fever and a high number of trophozoites in their blood count under the microscope. 
Enno et al. / GSC Biological and Pharmaceutical Sciences 2018, 02(02), 006-017

Table1 Composition within ATC Malachlorite $®$ tablet given to patients for treatment of parasite infection with Plasmodium falciparum

\begin{tabular}{|c|c|c|c|}
\hline $\begin{array}{l}\text { Sr. } \\
\text { No. }\end{array}$ & Composition & Content in mg & Role in treatment \\
\hline 1 & Sodium bicabonate & 44.0 & $\begin{array}{l}\text { To get an effervescent formulation, at the same time being a } \\
\text { penetration enhancer through mucous membranes of oral cavity }\end{array}$ \\
\hline 2 & Sodium chlorite & 15.0 & The active ingredient which later is converted into $\mathrm{ClO} 2$ \\
\hline 3 & Arabic gum & 197.9 & $\begin{array}{l}\text { An important agent to increase solubilization and absorption } \\
\text { through the mucosal lining }\end{array}$ \\
\hline 4 & Povidon 25 & 7.0 & $\begin{array}{l}\text { A synthetic polymer vehicle for dispersing \& suspending drugs } \\
\text { and also a binder for tablets }\end{array}$ \\
\hline 5 & Xylitab & 376.0 & A natural sweetener to increase tastiness \\
\hline 6 & Acesulfame-Potassium & 7.0 & $\begin{array}{l}\text { An artificial sweetener and also an enhancing agent for } \\
\text { absorption }\end{array}$ \\
\hline 7 & Prosol & 30.0 & $\begin{array}{l}\text { Silicified microcrystalline cellulose, combination of } \\
\text { microcrystalline cellulose (MCC) and colloidal silicon dioxide } \\
\text { (CSD) for binding properties of the tablet }\end{array}$ \\
\hline 8 & Citric acid $(540+110)$ & 65.0 & rmation of chlorine dioxide \\
\hline 9 & $\begin{array}{l}\text { Magnesium stearate } \\
40 \%\end{array}$ & 50.0 & $\begin{array}{l}\text { An inert compound for reducing stickiness during the } \\
\text { production process }\end{array}$ \\
\hline 10 & Artemisin & 1.9 & $\begin{array}{l}\text { An extract of Artemisia annua with oxygen radical scavenging } \\
\text { properties, an antinflammatory and an enhancer in efficacy of } \\
\text { antimalarial agents }\end{array}$ \\
\hline
\end{tabular}

The production of ACT Malachlorite ${ }^{\circledR}$ tablets followed good standard pharmaceutical practices (GSP) at an officially accredited and authorized laboratory in Switzerland by the name of Merisana ${ }^{\circledR}$ Company, in Cotébert, Switzerland..

\subsection{Dosing, inclusion, exclusion criteria, and drop outs}

A total dose of $75 \mathrm{mg}$ of sodium chlorite within 5 ACT Malachlorite ${ }^{\circledR}$ tablets was given 3 times per day with an interval of 8 hours. Starting off with five tablets on the first day this was followed by two times 3 tablets $(=45$ mg of sodium chlorite) at an interval of 8 hours over the following 5 days. Patients were instructed to place the tablet under their tongue (sublingual) and allow it to dissolve for about 10-15 minutes. The remnants of the sublingual tablet that remained in the buccal cavity was swallowed by the patient drinking a glass of water, not expecting any kind of residual effect. The patients were not allowed to suck, chew or swallow the tablet as this would have resulted in lower plasma concentrations of chlorine dioxide.

All doses of medication were administered under the supervision of a qualified member of the staff designated by the principal doctor on site of various health care facilities within the rural country of Cameroon. The study patients were observed for $30 \mathrm{~min}$ after administration for any kind of adverse reactions or vomiting. Any patient who vomited during this observation period was re-treated with the same dose of the medication and observed for an additional 30 min. If the patient vomited again, he or she was withdrawn and offered a rescue therapy. The patients were advised to stay in the health facility until they completed the treatment course for malaria infection with ACT Malachlorite®.

\subsection{Rescue medication in case of incompatibility}

If a patient vomited twice during the treatment phase he/she received parenteral therapy based on national treatment guidelines (IV saline, electrolytes etc.). Once a patient did not tolerate the trial medication he/she discontinued the treatment and an alternative anti-malaria medication was started. In this case, the reason for discontinuation was recorded in the case record form (CRF) as "Adverse Reaction" and the patient was withdrawn from the study and received parenteral therapy with Artemether or Quinine 7 days (IV) plus Tetracycline for 7 days as well as relevant supportive treatments. 
If a patient met one of the criteria for a therapeutic failure, he/she received Artemether at day $1 \mathrm{using} 3.2 \mathrm{mg} / \mathrm{kg}(\mathrm{max}$ $160 \mathrm{mg}$ ) intramuscularly, on day $2-1.6 \mathrm{mg} / \mathrm{kg}$ (max $80 \mathrm{mg}$ ) IM, and from day 3 to day $5-1.6 \mathrm{mg} / \mathrm{kg}$ (max $80 \mathrm{mg}$ ) per day intramuscularly. At day 6 Mefloquine $25 \mathrm{mg} / \mathrm{kg}$ was added which was spread over 3 days (max $1250 \mathrm{mg}$ ) according to the current national recommendation guidelines.

\subsection{Parameters measured for demonstration of efficacy}

Valid study end-points included either treatment failure, or completion of the follow-up period without treatment failure reflected in an adequate clinical and parasitological response, loss to follow-up, withdrawal from the study, and/or protocol violation. At all times, well-being of the patient had a priority over his or her continuation within the study.

Treatment outcomes were classified on the basis of an assessment of the parasitological and clinical outcome of the antimalarial treatment using the latest WHO guidelines [6]. Since elimination from the plasmodium parasites was the goal of the antimalarial therapy, all patients within the study who did show a treatment failure (non-effectiveness) were given a rescue medication with a follow-up until recovery. Also, the incidence of any adverse event was documented. All patients were asked routinely about previous symptoms and about symptoms that had emerged since the previous follow-up visit.

\subsection{Informed consent}

A standard physical examination was performed at baseline (day 0 before dosing) and on days 1, 2, 3, 4 and 6. Also, complete medical history including prior and concomitant medication, demographic data and contact details were taken at baseline.

Patients were only included in the study if adults and children (parents or guardians of children) had given their informed consent. The principal investigator(s) also obtained and documented the assent of children between the age of 12 and 18 years, but their assent had to be accompanied by the consent of a parent or a guardian. A child aged between 12 and 18 years who did not agree to participate was not enrolled in the study and was referred to the health facility staff to be treated according to the standard of care established by the Ministry of Health of Cameroon. Also, a written consent statement for the pregnancy test deemed necessary since a pregnant participant was not eligible to participate in the study. In addition, the need for contraception was required for female participants of child-bearing age who were sexually active.

\subsection{Patients inclusion and exclusion criteria}

The following criteria have to be met in order for a patient to participate in the study while on the other hand patients were excluded from the study as outlined in the following once he or she patient did not meet the criteria for enrollment or did not want to comply the conditions

\subsubsection{Inclusion criteria of patients to participate in the study}

i. An age between 10 and 60 years of age;

ii. A mono-infection with P.falciparum;

iii. The presence of an axillary temperature $\geq 37.5^{\circ} \mathrm{C}$ or a history of fever during the past $24 \mathrm{~h}$;

iv. The ability to swallow oral [by mouth] medication;

v. The ability and willingness to comply with the study protocol for the duration of the study and to comply with the study visit schedule;

vi. Signing of an informed consent by the patient or by a parent or guardian in the case of children aged less than 12 years;

vii. An informed assent from any participant aged more than 12 years and less than 60 years; and

viii. A consent for pregnancy testing from females of child-bearing age or from their parent or guardian if under 18 years.

\subsubsection{Exclusion criteria of patients not to participate in the study}

i. The presence of signs of severe falciparum malaria in children aged less than 10 years of age, according to the definition of the WHO

ii. An age less than 10 years of age;

iii. A mixed or mono-infection with another plasmodium species being detected by microscopy; 
iv. The presence of severe malnutrition (defined as a child whose growth standard is below $-3 \mathrm{z}$-score, has symmetrical edema involving at least the feet or has a mid-upper arm circumference of $<110 \mathrm{~mm}$ );

v. A febrile condition due to any kind of disease other than malaria (e.g. measles, acute lower respiratory tract infection, severe diarrhea with dehydration due to intestinal parasites, viral, or protozoa infection) or other known underlying chronic or severe diseases (e.g. cardiac, renal and hepatic disease, HIV/AIDS);

vi. Any regular medication which may have interfered with antimalarial pharmacodynamics and its kinetics;

vii. A history of a hypersensitivity reaction or a contraindication to the medication being tested or used as a rescue medication(s);

viii. All unmarried women 12-18 years of age with

ix. Being unable to or unwilling to take a pregnancy test or unwilling to take contraceptives for women of childbearing age.

\subsection{Parasite count before and during therapy with ACT Malachlorite ${ }^{\circledR}$}

Thick and thin blood films for parasite counts were obtained and examined at screening on day 0 to confirm adherence to the inclusion and exclusion criteria. Thick blood films were examined every once every 12 hours on day $1,2,3,4$ and 6 , once the patient returned spontaneously and parasitological reassessment was required.

Giemsa-stained thick and thin blood films were examined at a magnification of 1000 times to identify the parasite species and to determine parasite density. Three blood slides per patient were obtained consisting of two thick blood smears and one thin blood smear. One of the slides was stained immediately (10\% Giemsa for 10-15 min) for initial screening, while the others was kept for possible later screening and stored in a refrigerator.

For initial screening the thick blood smear was used to count the numbers of asexual parasites and white blood cells within a circumscribed microscopic area (number of parasites $/ \mathrm{mm}^{3}$ ). Parasitemia was defined once at least one parasite for every 16 white blood cells was found, corresponding to approximately 500 asexual parasites per microliter, reflecting a low-to-moderate parasitemia.

The second blood smear was used to calculate parasite density, by counting the number of asexual parasites in a set number of white blood cells (typically 200) with a hand tally counter. Once a field was started for calculation, it had to be counted until completion. Parasite density, was defined as the number of asexual parasites per $\mu$ l of blood. It was calculated by dividing the number of asexual parasites by the number of counted white blood cells and then multiplied by an assumed white blood cell density (typically 6000 per $\mu \mathrm{l}$ ) using the following equation.

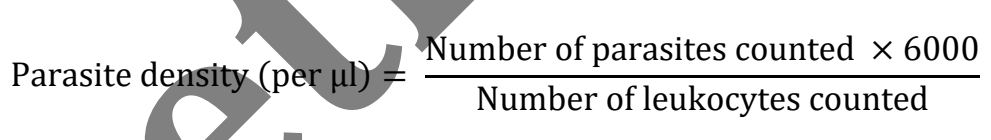

The same technique was used to establish parasite count on each subsequent blood film. When the number of asexual parasites was less than 100 per 200 white blood cells in any follow-up smear, counting had to be done again using a field containing at least 500 white blood cells (i.e. using a new field with at least 500 white blood cells).

A blood slide was considered negative when examination of 1000 white blood cells or 100 fields containing at least 10 white blood cells per field revealed no asexual parasites. Presence of gametocytes in a blood smear or on a follow-up slide were noted- however, this information was not used for basic calculation.

In addition, 100 fields of the second thick blood film were examined to exclude mixed infections on day 0; in case of doubt, the thin film was reexamined on a later time for confirmation purposes. If the examination of the thin film was not conclusive, the patient was excluded from the analysis and the following complete treatment regime within the follow-up period.

Two qualified microscopists read all the slides independently, while parasite densities were calculated by averaging the two counts. Blood smears with discordant results (differences between the two microscopists in species diagnosis, in parasite density of $>50 \%$ or in the presence of parasites) were re-examined by a third and independent microscopist, and parasite density were calculated by averaging the two closest counts.

\subsection{Statistical Analysis}

Failure of treatment to ACT Malachlorite $₫$ was considered positive if a decline in parasite count was not within the area of $80 \%$. Thus, the study power is $85 \%$ which is powerful enough to detect $20 \%$ difference between efficacy and non- 
efficacy within the one-sided treatment arm. The total sample size for the one-sided study arm was 500 with the adjustment of the recruitment period (one month and a half) and the duration of the proposed study 12 months. Due to the sample size with $P$. falciparum, statistical power exponential is 0.70 to 0.90 , resulting in an overall power of 0.85 during the period of 12 with a frequency period of 1.5 .

In case of drop-out or withdrawal, this patient was replaced by the next one in order to reassure that at the end of the study a minimum of 500 patients had completed the treatment phase. In addition to the reasons for withdrawal listed above, patients were considered withdrawn from the analysis if the decline in parasite count was unclassifiable or if the results of the analysis indicated that failure was due to a reinfection with $P$. falciparum.

For statically evaluation of efficacy the number of trophozoites within the treatment period were compared to baseline using the Wilcoxon matched-pair signed rank test. Significance is defined once $\mathrm{p}<0.05$. Before study initiation, official approval for conducting the study was obtained from the National Ethical Committee at the Institute of Public Health in Cameroon. The study key information will be posted on a public clinical trial registry (http://www.ANZCTR.org.au).

\section{Results}

Of the 579 patients recruited for the study 79 dropped out or were excluded from the analysis because of different reasons. Of these dropouts 29 did not show up for the next evaluation, 30 dropped out because of violation not adhering to the study protocol and 10 were misdiagnosed as they had fever of different origin. In addition, the two youngest patients in the group 1-4 years of age (table 2) were not included in the study analysis because they had additional infections which needed special medical treatment in the neonatal care unit. All other 500 adhered to the protocol and they were used for the analysis demonstrating the effectiveness of ATC Malachlorite ${ }^{\circledR}$ in the treatment of malaria. Although ATC Malachlorite ${ }^{\circledR}$ tablets were given to patients of different sex and age who all lived in the rural area, there were not differences in side effects attributing them to gender difference (table 2).

Table 2 Summary of demographic data of patients taking part in the study using ATC Malachlorite $®$ tablets and the incidence of side-effects during the treatment of malaria infection $(n=500$; mean $+/-\mathrm{SD})$

\begin{tabular}{|c|c|c|c|c|}
\hline & $1-4$ & & $21-50$ & $>50$ \\
\hline \multicolumn{5}{|l|}{ Parameters } \\
\hline Number of patients & 2 & & 240 & 80 \\
\hline \multirow{2}{*}{$\begin{array}{ll} & \text { Male } \\
\text { Sex } & \text { Female }\end{array}$} & 2 & & 100 & 60 \\
\hline & & 100 & 140 & 20 \\
\hline Body weight (kg) & 5 & $65 \pm 7$ & $61 \pm 8$ & $55 \pm 5$ \\
\hline Height $(\mathrm{cm})$ & & $171 \pm 9$ & $175 \pm 9$ & $160 \pm 7$ \\
\hline Side effects & $\begin{array}{l}\text { Gastric upset with } \\
\text { painful cramps }\end{array}$ & $\begin{array}{l}\text { Unpleasant pungent } \\
\text { chlorine taste }\end{array}$ & $\begin{array}{l}\text { Abdominal discomfort, } \\
\text { cramping bloating }\end{array}$ & Chlorine taste \\
\hline Severity* & +++ & ++ & ++ & + \\
\hline
\end{tabular}

All patients included within the study showed acute symptoms of varying degrees affecting the whole person, showing a close correlation of parasite blood count done at the first visit by their local doctor. Symptoms included undulant fever, joint pain, throbbing headache, loss of appetite, muscle aches and general weakness.

Demographic data revealed that the majority of patients were in the middle age group (21-50 years), as depicted in Table 2.

With a mean number of 6430 (+/- 816 SD) of trophozoites per $\mathrm{mm}^{3}$ reflects a high infection rate also explaining the severity of their symptoms. Following the intake of ATC Malachlorite $₫$, blood count of Plasmodium falciparum significantly declined $(\mathrm{p}<0.001)$ to a mean of $4932(+/-842$ SD) trophozoites on day two and further declined to 1454 $(+/-401$ SD; $p<0.001)$ on day four, while no trophozoites could be detected in the blood on day six (Fig. 1). Every count 
demonstrated a highly significant decline to the previous count $(\mathrm{p}<0.001)$ suggesting high efficacy in the use of ATC Malachlorite $\circledR$ for the eradication of Plasmodium falciparum in malaria patients.

Parallel to the decline of microbes, patients quickly recovered regaining their well-being and on day six no pre-existing symptoms were reported by patients. But apart from the quick recovery of acute malaria infection, it should be noted that none of the patients suffered extreme side effects nor abandoned the cycle of medication due to an intolerance of ATC Malachlorite ${ }^{\circledR}$ medication.

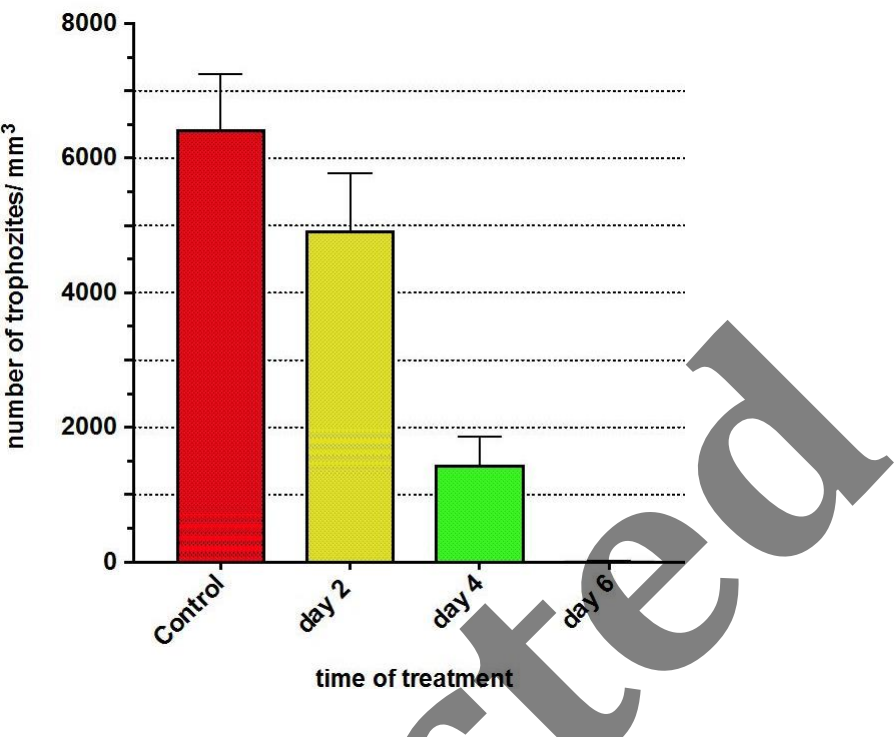

Figure 1 Results of treatment with ATC Malachlorite $®$ tablets in patients with acute malaria infection, as evidenced by the number of trophozoites $/ \mathrm{mm} 3$ in the blood samples (sample size $\mathrm{n}=500$; mean \pm SD)

\section{Discussion}

This so far is the first publication of data on patients with infection of Plasmodium falciparum who were treated with the active ingredient chlorine dioxide $\left(\mathrm{ClO}_{2}\right)$ plus artemisin, demonstrating a highly significant reduction in parasite count while at the same time resulting in marked reduction of their clinical symptoms. The efficacy of anti-malarial principle of chlorine dioxide, as demonstrated in the present pilot study, is due to killing of all asexual blood stages of parasites that are responsible for the disease manifestations (schizontic activity of the blood). The data also suggests that the antimalarial effect involves lysosomal trapping in the intra-erythrocytic parasite, followed by binding to the toxic hemin which is produced during the digestion of hemoglobin [7] and where heme acts as an oxidant sensitizer of chlorine dioxide $[8,9]$.

As to the mode of action, sodium chlorite $\left(\mathrm{NaCl}_{2}\right)$ presents a totally new avenue in the treatment of this disease as it inherits a number of advantages. Use of the prodrug sodium chlorite $\left(\mathrm{NaCl}_{2}\right)$ compressed together with dry powdered citric acid in a readily soluble tablet, which only when getting into contact with water, saliva or gastric acid, results in the formation of chlorine dioxide $\left(\mathrm{ClO}_{2}\right)$. After being taken up by the mucous membranes and getting into the circulation, due its negative charge chlorine dioxide selectively attaches to the positively charged plasmodium parasite, thus releasing a highly aggressive, unpaired oxygen radical $\left(\mathrm{O}_{2}^{-}\right)$. And in order to complete itself, the oxygen radical tears out electrons from the outside layer of the plasmodium, inducing a disruption and a killing of the parasite. Therefore, the mode of action of chlorine dioxide is based on the formation of the oxygen radical, disrupting the outside layer of the plasmodium, initiating a chain reaction which ultimately results in a disintegration of the whole parasite turning its inside to the outside.

While it is well known that chloride dioxide can quickly kill many types of bacteria [10-11] and protozoa such as the asexual blood stages of Plasmodium falciparum that are responsible for disease manifestation (schizontic activity of blood) in malaria [12-15] and Plasmodium vivax, Plasmodium falciparum, Plasmodium ovale and Plasmodium malariae just like bacteria, are indeed sensitive to oxidants like chlorine dioxide resulting in a quick destruction of Plasmodium falciparum and $P$. vivax, including strains resistant to multiple drugs. Since free radicals from ACT Malachlorite ${ }^{\circ}$ are responsible for killing malaria parasites, the selectivity of their toxicity is likely related to the fact they reach high concentrations in the human body in infected red blood cells while at the same time attacking selectively the plasmodia. This is because they are especially sensitive to oxidants (electron grabbers) and upon sufficient removal of the parasite's 
life sustaining thiols by oxidation, the parasite rapidly dies [16-20]. Such a connotation is underlined by the current data where a rapid decline of parasites in the blood is observed. And once the concentration remains high for at least three to four days, there is little chance for any other parasite to escape this deadly force of $\mathrm{ClO}_{2}$. This assumption is also underlined by data from other areas in human life, where chlorine dioxide is used for the decontamination of drinking water getting rid of residual virus, bacteria or protozoa [18, 21-24] a reason, why it is important that high blood concentrations appear to be more effective than all other known antimalarial drugs in use since this day.

While it was estimated that treatment with conventional medications reduces the parasite biomass by a factor of about 10 on each asexual cycle of the parasite from 36 to 48 hours and by a factor of 106-108 on a 3-day course treatment, the current findings indicate a much higher efficacy by a factor of almost $100 \%$ over a period of three hour, it is believed that chlorine dioxide, like quinine, very much unlikely results in a tolerance development, which so far always has been the major obstacle in fighting malaria in the long run.

There is less information about the effect against the other two human malaria parasites, Plasmodium malariae and Plasmodium ovale, but they are likely to be attacked in a similar way [25-32]. Moreover, it is known that chlorine dioxide has a certain effect on gametocytes, a stage which is infectious for mosquitoes ingesting a blood meal from an infected person [7]. What is also known from animal studies, that $\mathrm{ClO}_{2}$ has effect on hypnozoites which are in the liver and can cause relapses in Plasmodium vivax and Plasmodium ovale malaria [33]; however, conclusive evidence of such effects in human is still lacking.

Biochemical studies suggest that the antimalarial effect of oxygen radicals from hydrogen peroxide as well as from chlorine dioxide involves lysosomal entrapment of the drug in the intra-erythrocytic parasite followed by the link to the toxic hemin which is produced during the digestion of hemoglobin [34-35]. This binding prevents polymerization of nontoxic hemin pigment of malaria [36]. The mechanism of action differs from that of any other anti-malaria drug and is closely related to the release of oxygen free radicals. Such a release appears at a peroxide bridge which bursts within the molecule and occurs when the compound meets high levels of heme, a hemoglobin degradation product produced by malaria parasites living in red blood cells [37]. In living things including parasites, iron is a necessary cofactor for many enzymes. Thus it is reasonable to estimate that any damage to plasmodia caused by oxides of chlorine is compounded by conversion of ferrous $(\mathrm{Fe}++)$ cofactors to ferric $(\mathrm{Fe}+++)$ [38-39]. All these events occur within three to four days of treatment as demonstrated in this present set of patients.

As for the pharmacokinetics, the rapid absorption and the large volumes of distribution, together with a short half-live are two main characteristically traits of chlorine dioxide [40]. Since it also penetrates the blood-brain-barrier and accumulates in erythrocytes it explains why there is an immediate effect also related to malaria induced CNS symptoms [41].

Despite the potential neurotoxicity of chlorine dioxide, which has been demonstrated in animal studies at doses that far exceed those used in the present treatment of malaria in patients, no such side-effects were observed in the present set of patients. Also, animal studies using much higher oral or topical doses have proven relatively safety [42-43], so it is not unexpected that we did not encounter any serious side effects. This is also underlined by others who had demonstrated no serious side effects in humans when chlorine dioxide is being used for water decontamination [44].

The potential side effects of treatment with chlorine dioxide used for water disinfection, as reported in the literature at even high therapeutic doses are rare, and mostly involve the formation of methemoglobin [42, 45], with a low incidence of dizziness, fatigue and palpitations, while myalgia, sleep disorders are practically absent, and joint pain, headaches and rashes can be related to a dysbalance of gut bacteria [46]. The latter can be expected especially when part of the sodium chlorite is being swallowed and where the newly formed oxygen radical results in the elimination of beneficial gut bacteria which effects the response of the immune system being located by up to $80 \%$ within the gut [47]. In this context, it is important to note that many the above mentioned side effects as reported in the literature, have not been observed in our series of patients and when the medication was taken according to instructions. Especially, and in contrast to the present available combination therapy for the treatment of malaria, ACT Malachlorite® is less expensive in manufacturing, it has demonstrated practically no serious side effects and it has a high acceptance in patients and showed a significant therapeutic efficacy after 3 to 4 days of treatment. Therefore, it can be recommended as a first-line routine treatment in countries where resistance to chloroquine or agents of the newer generation containing artemisinin present a problem, especially in countries with endemic malaria such as Cameroon, Gambia or the south of Senegal where on site visits of health care personal in the rural area are rare and patients often, even once diagnosed with malaria, are left unattended. Since there is already a resistance against sulfadoxinel pyrimethamine, the useful life of a combination therapy is limited life quality of a combination therapy is very much limited. 


\section{Conclusion}

Higher priority should be given to the search for alternative methods in malaria treatment today. This demand, which had realized in the present study especially as scientists have recently been warning of a "supermalaria" parasite that is quickly spreading throughout Southeast Asia and will pose a worldwide health threat if it makes its way to Africa. This parasite is resistant to the most common first-line malaria treatment, artemisinin [48]. Also, the high level of genetic diversity between the different species of mosquitoes and their ability to swap their genes is making it very difficult to prevent insecticide-resistant groups from forming. While new insecticides are being developed - such as those that kill mosquitoes with genetic approaches, stop them from breeding or prevent them from transmitting the parasite responsible for malaria - experts are worried that the mosquitoes will simply develop resistance to these methods as well. According to the World Health Organization, the gains seen in malaria prevention in recent years are under threat from growing insecticide resistance that could ultimately lead to a significant rise in malaria cases and deaths. They've called on the global malaria community to act urgently to keep insecticide resistance under control. 212 million people a year are infected with malaria, and children are at particular risk of dying from it [49]. Since 2010, 61 countries have reported mosquitoes developing resistance to one or more insecticide class, and 50 of those countries report on mosquitoes developing resistance to two or more classes. The problem could actually be worse than the statistics indicate as many countries are not reporting their data quickly or are failing to monitor for insecticide resistance on a routine basis. In addition, drug resistance making the problem even worse. This parasite is resistancy is evident to the most common first-line malaria treatment, artemisinin [50]. With 438,000 malaria deaths around the world in 2015, it's frightening to think of what kind of mortality figures a super malaria could cause.

In summary, this new sublingual ATC Malachlorite ${ }^{\circledR}$ tablet formulation presents a promising new approach in malaria treatment as it is based on a completely new mode of action. It proved to be safe and effective and should be introduced to replace the combination therapy such as artesunate plus sulfadoxinel pyrimethamine. The replacement would very likely allow a longer useful life, because so far there is no reported resistance to the components in ATC Malachlorite ${ }^{\circ}$. And finally, the wide distribution as a package, such as a blister (blister pack) where only one component is kept, improves patient compliance, while newer fixed combination formulations would take more years to develop.

\section{Compliance with ethical standards}

\section{Acknowledgments}

The work and assistance of all doctors and nurses in the rural areas of Cameroon who had participated in the study and who by means of their meticulous data acquisition is gratefully acknowledged, making the study a valuable contribution in the treatment of malaria.

\section{Disclosure of conflict of interest}

The authors of the paper declare that there is no conflict of interest, that they have seen and approved the manuscript being submitted and that the paper has not been published in total or in part elsewhere. Also, the authors listed on the title page attest that they have signifieantly contributed to the work have read the manuscript, attest to the validity and the legitimacy of the data and its interpretation and agree for the submission to the journal. All authors agree that the authors list is correct in its content and order and no modification of the authors list will be made.

\section{Statement of ethical approval}

Before study initiation, official approval for conducting the study was obtained from the National Ethical Committee at the Institute of Public Health in Cameroon.

\section{Statement of informed consent}

Patients were only included in the study if adults and children (parents or guardians of children) had given their informed consent. The principal investigator(s) also obtained and documented the assent of children between the age of 12 and 18 years, but their assent had to be accompanied by the consent of a parent or a guardian. 


\section{References}

[1] Tatem AJ, Jia P, Ordanovich D, Falkner M, Huang Z, Howes R, Hay SI, Gething PW and Smith DL. (2017). The geography of imported malaria to non-endemic countries: a meta-analysis of nationally reported statistics. The Lancet Infectious Diseases, 17(1), 98-107.

[2] Basco LK, Ndounga M, Keundjian A and Ringwald P. (2002). Molecular epidemiology of malaria in cameroon. IX. Characteristics of recrudescent and persistent Plasmodium falciparum infections after chloroquine or amodiaquine treatment in children. The American journal of tropical medicine and hygiene, 66(2), 117-23.

[3] Maitland K, Bejon P and Newton, CRJC. (2003). Malaria. Current Opinion in Infectious Diseases, 16, 389-395.

[4] James S and Miller L. (2003). Malaria vaccine development status report. In Doherty AM (Ed), Annual Reports in Medicinal Chemistry, Academic Press, New York, 203-211.

[5] World Health Organization. (2012). WHO position statement on effectiveness of non-pharmaceutical forms of Artemisia annua L. against malaria. WHO Press, Geneva, 4.

[6] Ringwald P, Shallcross L, Miller JM, Seiber E and World Health Organization. (2005). Susceptibility of Plasmodium falciparum to antimalarial drugs: report on global monitoring 1996-2004, WHO Press, Geneva.

[7] Jefford CW. (2001). Why artemisinin and certain synthetic peroxides are potent antimalarial- implications for the mode of action. Current medicinal chemistry, 8(15), 1803-1826.

[8] Francis SE, Sullivan Jr DJ and Goldberg ADE. (1997). Hemoglobin metabolism in the malaria parasite Plasmodium falciparum. Annual Reviews in Microbiology, 51(1), 97-123.

[9] Saliba KJ, Allen RJ, Zissis S, Bray PG, Ward SA and Kirk K. (2003). Acidification of the malaria parasite's digestive vacuole by a H+-ATPase and a H+-pyrophosphatase. Journal of Biological Chemistry, 278(8), 5605-5612.

[10] Young SB and Setlow P. (2003). Mechanisms of killing of Bacillus subtilis spores by hypochlorite and chlorine dioxide. Journal of Applied Microbiology, 95(1), 54-67.

[11] Harakeh S, Illescas A and Matin A. (1988). Inactivation of bacteria by purogene. Journal of Applied Microbiology, 64(5), 459-463.

[12] Winiecka-Krusnell, J and Linder E. (1998). Cystícidal effect of chlorine dioxide on Giardia intestinalis cysts. Acta tropica, 70(3), 369-372.

[13] Korich DG, Mead JR, Madore MS, Sinclair NA and Sterling CR. (1990). Effects of ozone, chlorine dioxide, chlorine, and monochloramine on Cryptosporidium parvum oocyst viability. Applied and environmental microbiology, 56(5), 1423-1428.

[14] LeChevallier MW, Au KK. (2004).Water treatment and pathogen control. Iwa Publishing, Geneva, 52-54.

[15] Driedger AM, Rennecker JL and Mariñas BJ. (2000). Sequential inactivation of Cryptosporidium parvum oocysts with ozone and free chlorine. Water Research, 34(14), 3591-3597.

[16] Müller S, Gilberger TW, Krnajski Z, Lüersen K, Meierjohann S and Walter RD. (2001). Thioredoxin and glutathione system of malaria parasite Plasmodium falciparum. Protoplasma, 217(1-3), 43-49.

[17] Rahlfs S, Nickel C, Deponte M, Schirmer RH and Becker K. (2003). Plasmodium falciparum thioredoxins and glutaredoxins as central players in redox metabolism. Redox report, 8(5), 246-250.

[18] Jaeger T and Flohe L. (2006). The thiol-based redox networks of pathogens: unexploited targets in the search for new drugs. Biofactors, 27(1-4), 109-120.

[19] Olagunju O, Siegel PD, Olojo R and Simoyi RH. (2006). Oxyhalogen- sulfur chemistry: kinetics and mechanism of oxidation of N-Acetylthiourea by chlorite and chlorine dioxide. The Journal of Physical Chemistry A, 110(7), 2396-2410.

[20] Müller S. (2004). Redox and antioxidant systems of the malaria parasite Plasmodium falciparum. Molecular microbiology, 53(5), 1291-1305.

[21] Dowling LT. (1974). Chlorine dioxide in potable water treatment. Water Treatment and Examination, 23(2), 190204.

[22] Granstrom ML and Lee GF. (1958). Generation and use of chlorine dioxide in water treatment. Journal (American Water Works Association), 50(11), 1453-1466. 
[23] Augenstein H. (1968). Use of chlorine dioxide to disinfect water supplies. Journal - American Water Works Association, 60(939).

[24] LeChevallier MW and Au . (2004). Water treatment and pathogen control in process efficiency in achieving safe drinking water, world health organization, editor, IWA Publishing, Geneva. p. 52-54.

[25] Biot C, Dessolin J, Grellier P and Davioud-Charvet E. (2003). Double-drug development against antioxidant enzymes from Plasmodium falciparum. Redox report, 8(5), 280-283.

[26] Turrens JF. (2004). Oxidative stress and antioxidant defenses: a target for the treatment of diseases caused by parasitic protozoa. Molecular aspects of medicine, 25(1-2), 211-220.

[27] Oliveira PL and Oliveira MF. (2002). Vampires, Pasteur and reactive oxygen species. FEBS letters, 525(1-3), 3-6.

[28] Allison AC and Eugui EM. (1983). The role of cell-mediated immune responses in resistance to malaria, with special reference to oxidant stress. Annual review of immunology, 1(1), 361-388.

[29] Vennerstrom JL. (1989). Amine peroxides as potential antimalarials. Journal of medicinal chemistry, 32(1), 6467.

[30] Senok AC, Nelson EA, Li K, Oppenheimer SJ. (1997). Thalassaemia trait, red blood cell age and oxidant stress: effects on Plasmodium falciparum growth and sensitivity to artemisinin. Transactions of the Royal Society of Tropical Medicine and Hygiene, 91(5), 585-589.

[31] Dockrell HM and Playfair JH. (1984). Killing of Plasmodium yoelii by enzyme-induced products of the oxidative burst. Infection and immunity, 43(2), 451-456.

[32] Clark IA, Butcher GA, Buffinton GD, Hunt NH, Cowden WB. (1987). Toxicity of certain products of lipid peroxidation to the human malaria parasite Plasmodium falciparum. Biochemical pharmacology, 36(4), 543-546.

[33] Siddiqi NJ and Pandey VC. (1999). Studies on hepatic oxidative stress and antioxidant defense systems during arteether treatment of Plasmodium yoelii nigeriensis infected mice. In Stress Adaptation, Prophylaxis and Treatment, Springer, Boston, 169-173.

[34] Mishra NC, Kabilan L and Sharma A. (1994). Oxidative stress and malaria-infected erythrocytes. Indian journal of malariology, 31(2), 77-87.

[35] Dockrell HM and Playfair JH. (1983). Killing of blood-stage murine malaria parasites by hydrogen peroxide. Infection and immunity, 39(1), 456-459.

[36] Clark IA and Hunt NH. (1983). Evidence for reactive oxygen intermediates causing hemolysis and parasite death in malaria. Infection and Immunity, 39(1), 1-6.

[37] Rosenthal PJ and Meshnick SR. (1996). Hemoglobin catabolism and iron utilization by malaria parasites. Molecular and biochemical parasitology, 83(2), 131-139.

[38] Cabantchik I, Glickstein H, Golenser J, Loyevsky M and Tsafack A. (1996). Iron chelators: mode of action as antimalarials. Acta haematologica, 95(1), 70-77.

[39] Padmanaban G and Rangarajan PN. (2000). Heme metabolism of plasmodium is a major antimalarial target. Biochemical and biophysical research communications, 268(3), 665-668.

[40] Abdel-Rahman MS, Couri D and Bull RJ. (1979). Kinetics of Cl02 and effects of Cl02, Cl02-, and Cl03-in drinking water on blood glutathione and hemolysis in rat and chicken. Journal of environmental pathology and toxicology, 3(1-2), 431-449.

[41] Toth GP, Long RE, Mills TS and Smith MK. (1990). Effects of chlorine dioxide on the developing rat brain. Journal of Toxicology and Environmental Health, Part A Current Issues, 31(1), 29-44.

[42] Moore G and Calabrese EJ. (1980). The effects of chlorine dioxide and sodium chlorite on erythrocytes of A/J and C57L/J mice. Journal of environmental pathology and toxicology, 4(2-3), 513-524.

[43] Bercz JP, Jones L, Garner L, Murray D, Ludwig DA and Boston J. (1982). Subchronic toxicity of chlorine dioxide and related compounds in drinking water in the nonhuman primate. Environmental Health Perspectives, $46,47$.

[44] Heffernan WP, Guion C and Bull RJ. (1979). Oxidative damage to the erythrocyte induced by sodium chlorite, in vivo. Journal of environmental pathology and toxicology, 2(6), 1487-1499.

[45] Moore GS, Calabrese EJ, DiNardi SR and Tuthill RW. (1978). Potential health effects of chlorine dioxide as a disinfectant in potable water supplies. Medical hypotheses, 4(5), 481-496. 
[46] Martinez KB, Leone V and Chang EB. (2017). Western diets, gut dysbiosis, and metabolic diseases: Are they linked?. Gut microbes, 8(2), 130-142.

[47] Vighi G, Marcucci F, Sensi L, Di Cara G and Frati F. (2008). Allergy and the gastrointestinal system. Clinical and Experimental Immunology, 153(s1), 3-6.

[48] Fouet C, Atkinson P and Kamdem C. (2018). Human interventions: driving forces of mosquito evolution. Trends in parasitology.

[49] World Health Organization. (2017). Framework for a national plan for monitoring and management of insecticide resistance in malaria vectors. Malaria Vector Control Technical Expert Group of WHO, Editor, World Health Organization, Geneva, 39.

[50] Imwong M, Hien TT, Thuy-Nhien NT, Dondorp AM and White NJ. (2017). Spread of a single multidrug resistant malaria parasite lineage (PfPailin) to Vietnam. The Lancet Infectious Diseases, 17(10), 1022-1023.

\section{How to cite this article}

Enno F, Hans-Peter S and Olivia MW. (2018). ACT Malachlorite ${ }^{\circledR}$ for treatment of patients with acute Plasmodium falciparum infection: A pilot study incorporating 500 patients in the rural area of Cameroon. GSC Biological and Pharmaceutical Sciences, 2(2), 06-17. 